\title{
A cost effectiveness analysis within a randomised controlled trial of post-acute care of older people in a community hospital
}

\author{
Jacqueline O’Reilly, Karin Lowson, John Young, Anne Forster, John Green, Neil Small
}

\begin{abstract}
Objective To assess the cost effectiveness of post-acute care for older people in a locality based community hospital compared with a department for care of elderly people in a district general hospital, which admits patients aged over 76 years with acute medical conditions.

Design Cost effectiveness analysis within a randomised controlled trial.

Setting Community hospital and district general hospital in Yorkshire, England.

Participants 220 patients needing rehabilitation after an acute illness for which they required admission to hospital.

Interventions Multidisciplinary care in the district general hospital or prompt transfer to the community hospital.

Main outcome measures EuroQol EQ-5D scores transformed into quality adjusted life years (QALYs), and health and social service costs over six months from randomisation.

Results The mean QALY score for the community hospital group was marginally non-significantly higher than that for the district general hospital group $(0.38 v 0.35)$ at six months after recruitment. The mean (standard deviation) costs per patient of the health and social services resources used were similar for both groups: community hospital group $£ 7233$ (euros 10 567; \$13 341) (£5031), district general hospital group $£ 7351$ (£6229), and these findings were robust to several sensitivity analyses. The incremental cost effectiveness ratio for community hospital care dominated. A cost effectiveness acceptability curve, based on bootstrapped simulations, suggests that at a willingness to pay threshold of $£ 10000$ per QALY, $51 \%$ of community hospital cases will be cost effective, which rises to $53 \%$ of cases when the threshold is $£ 30000$ per QALY. Conclusion Post-acute care for older people in a locality based community hospital is of similar cost effectiveness to that of an elderly care department in a district general hospital.
\end{abstract}

\section{Introduction}

Demographic transition in the developed world is prompting governments to develop health and social care policies capable of effectively tackling the needs of older people. In the United Kingdom the role of primary care trusts as commissioners and their close working links with social care agencies for elderly people further underlines the importance of identifying outcome and cost data. ${ }^{1}$ The NHS Plan, a 10 year programme of health reform in the United Kingdom, heralded the introduction of intermediate care services for older people. ${ }^{2}$ Intermediate care encompasses several service models for which the evidence base of clinical and health economics is poorly developed. Three ran- domised controlled trials evaluating nurse led intermediate care wards reported similar clinical outcomes to usual care but found that lengths of hospital stay were significantly greater on the nurse led wards, therefore the associated costs were likely to be greater. $^{3-5}$ For "hospital at home" intermediate care services a meta-analysis of 16 randomised controlled trials also reported similar outcomes but at similar costs. ${ }^{6}$ The potential of community hospitals to provide intermediate care has been recognised, particularly because they comprise an existing and well developed healthcare resource. ${ }^{89}$ We carried out a cost effectiveness study within a randomised controlled trial of community hospital based intermediate care for older people in need of rehabilitation.

\section{Methods}

The community hospital studied has 18 beds and serves the population $(n=92300)$ of north Bradford primary care trust. The cost effectiveness study was embedded within a randomised controlled trial comparing clinical and service use outcomes between the community hospital and a department for the care of elderly people in a district general hospital. The trial methods and community hospital are described in detail elsewhere. ${ }^{10}$ Briefly, patients registered with a general practitioner in the primary care trust served by the community hospital and admitted as emergency referrals to the elderly care department in a district general hospital in Bradford were eligible for inclusion in the study once they had become medically stable and considered in need of post-acute rehabilitation by the responsible consultant. Consent was sought and patients were randomised in a ratio of one patient to remain in the district general hospital to two patients to be transferred to the community hospital. Recruitment to the study took place between November 2000 and September 2002. The economic evaluation adopted a whole systems approach, investigating the costs to both health and social care services over the six months after randomisation.

\section{Outcome measure}

We measured health outcomes at baseline, one week after discharge, and three and six months after randomisation using the European quality of life instrument EuroQol EQ-5D. As this measure is not disease specific it can be used to evaluate and compare the effect on health of a range of different interventions and diseases. ${ }^{11}$ On the assumption that no further change in the score occurred during the remainder of the year, we transformed the EuroQol scores into quality adjusted life years (QALYs). ${ }^{12}$

\section{Utilisation of resources}

We recorded use of resources one week after discharge and three and six months after randomisation, using a questionnaire 
administered by an interviewer to patients and their carers. The questionnaire collects information on several resource categories, including hospital admissions, attendances to accident and emergency departments, visits to the general practitioner and hospital outpatient departments, use of out of hours services, contact with health or social care staff, institutional accommodation (for example, placement in a care home), and receipt of aids and adaptations. In cases of missing data on resource use (where usage, but not frequency, was recorded on the questionnaire), we assumed that the frequency of use for that particular resource by the patient was equal to the mean for the treatment group. If no resource usage was indicated on the questionnaire then we assumed that this was correct. The primary source for information on hospital inpatient activity was the patient administration system. We included only non-elective hospital admissions. To investigate the reliability of patient and carer recall of use of community services (district nursing, chiropody, physiotherapy, occupational therapy), we checked the results of the first 40 patients against records held on a community database. We used the kappa statistic to investigate the extent of agreement.

\section{Costs}

We used a combination of local and national sources to calculate the health and social care costs for each patient over the six months after randomisation. Local information was used to calculate the costs of the initial length of hospital stay after recruitment with daily rates obtained from the finance department of the primary care trust responsible for the community hospital and from specialty costs obtained from the hospital trust's financial returns for the district general hospital. ${ }^{13}$ The daily rates for the community hospital include direct and indirect costs, with expenditure on support services and overheads of buildings as well as staffing costs. Staff costs include the nursing establishment, two consultant sessions per week, four hospital practitioner sessions per week, 0.5 whole time equivalent physiotherapist and occupational therapist, one whole time equivalent therapy helper, and one weekly session of speech and language and community dietician time. The daily costs of the hospitals were comparable for resource categories included. We have used full economic costs in both cases. The costs of hospital readmissions were based on trust specific costs for health resource group ${ }^{14}$ with additional daily hospital costs if the actual length of stay was greater than that expected from the trust specific health resource group. Costs for the community services used by the patients were obtained from the Personal and Social Services Research Unit. ${ }^{15}$

We calculated costs net of patient contributions when relevant. For community based services, such as chiropody and home care, we assumed that those patients who contributed to these services incurred the total cost of these visits. For those who contributed to the costs of institutional care, we assumed contributions as $30 \%$ of cost. As institutional care was a substantial component of costs, we tested this assumption in a sensitivity analysis. We obtained unit costs for most types of equipment from the NHS Purchasing and Supply Agency. Unit costs for other equipment were obtained from the websites of manufacturers and distributors and from the Personal Social Services Research Unit. ${ }^{15}$

To be consistent with the calculation of QALYs, we assumed that the only resources that patients used were those captured during the six months of follow-up. Therefore under this assumption patients did not incur any other costs during the remainder of the year. The price year was 2001-2 and the currency was UK pounds sterling. Since the timescale of the trial was under a year, it was not necessary to discount costs and health benefits.

\section{Analysis}

We adopted a cost effectiveness approach to the health economic analysis over the six months of the study using the intention to treat principle. We used $t$ tests to compare differences between the two groups for changes in the QALY scores from baseline to assessments. Differences between the groups in changes in QALYs from baseline were also compared using analysis of covariance to adjust for baseline factors (age, sex, patient in institutional care, mental status using abbreviated mental test score ${ }^{16}$ and disability using the Barthel index ${ }^{17}$ ). To investigate potential bias due to death, we repeated the analyses after excluding those patients who had died during the study.

Use of itemised resources over the study period is expressed as means (standard deviations). When sample size permitted we used $t$ tests to compare the groups. We calculated costs per patient by multiplying resource volumes by unit costs, and we used $t$ tests to compare the mean total costs for the groups. The incremental cost effectiveness ratio was calculated as the ratio of the differences between the groups for mean costs per patient and mean QALYs per patient. In calculating this ratio, we assumed patients with missing QALY scores to have scores equal to the mean patient in the treatment group. We undertook nonparametric bootstrapping on the incremental cost and effectiveness with 50000 replications. ${ }^{18}{ }^{19}$ A cost effectiveness acceptability curve was constructed.

\section{Results}

Overall 220 patients were randomised: 141 to care in a locality based community hospital and 79 to usual care in a district general hospital. Twenty six patients did not achieve their allocated group: 16 remained inappropriately in the district general hospital, and 10 were transferred inappropriately to the community hospital. In total, 72 patients were transferred within the intended two days after randomisation and 49 were transferred after more than two days. At the end of the six months' follow-up 55 patients had died: 34 assigned to the community hospital group and 21 assigned to the district general hospital group.

\section{Health outcomes}

The mean QALYs were marginally greater for the district general hospital group at baseline but were higher for the community hospital group at each assessment point (table 1). The between group differences for the changes in QALY scores from baseline to the assessment points were not statistically significant with (analysis of covariance $\mathrm{P}>0.34$ ) or without adjustment for baseline factors ( $t$ test $\mathrm{P}>0.28$ ). When patients who had died during the trial were excluded from the analysis, the average QALYs increased for both groups but the between group differences for the changes from baseline to the assessment points remained statistically non-significant $(t$ test $\mathrm{P}>0.77)$.

\section{Utilisation of resources and costs}

The groups had a similar mean length of hospital stay during the initial admission after randomisation: 22 days for the community hospital group and 23 days for the district general hospital group. The daily costs were also similar: $£ 148$ for the community hospital group and $£ 146$ for the district general hospital group. The proportions of patients using the resources listed in table 2, and the mean quantity of resources used, were similar for both groups. The reliability study indicated moderate agreement over 
Table 1 Baseline, follow-up, and between group differences in quality adjusted life years for older patients admitted to a community hospital or district general hospital for rehabilitation

\begin{tabular}{|c|c|c|c|c|c|}
\hline \multirow{2}{*}{ Variable } & \multicolumn{2}{|c|}{ Community hospital } & \multicolumn{2}{|c|}{ District general hospital } & \multirow{2}{*}{$\begin{array}{l}\text { Mean }(95 \% \mathrm{Cl}) \text { between group difference: } \\
\text { change from baseline }\end{array}$} \\
\hline & No of patients & Mean (SD) & No of patients & Mean (SD) & \\
\hline \multicolumn{6}{|l|}{ All patients: } \\
\hline Baseline & 139 & $0.41(0.30)$ & 78 & $0.43(0.28)$ & - \\
\hline 1 week after discharge & 125 & $0.46(0.35)$ & 72 & $0.44(0.36)$ & $0.04(-0.06$ to 0.15$)$ \\
\hline 3 months after recruitment & 119 & $0.40(0.37)$ & 72 & $0.36(0.36)$ & $0.06(-0.06$ to 0.18$)$ \\
\hline 6 months after recruitment & 125 & $0.38(0.34)$ & 68 & $0.35(0.34)$ & $0.06(-0.05$ to 0.18$)$ \\
\hline \multicolumn{6}{|l|}{ Surviving patients*: } \\
\hline Baseline & 105 & $0.45(0.30)$ & 57 & $0.43(0.29)$ & - \\
\hline 1 week after discharge & 94 & $0.57(0.30)$ & 53 & $0.53(0.33)$ & $0.02(-0.10$ to 0.13$)$ \\
\hline 3 months after recruitment & 89 & $0.53(0.33)$ & 52 & $0.49(0.34)$ & $0.01 \quad(-0.12$ to 0.14$)$ \\
\hline 6 months after recruitment & 91 & $0.52(0.30)$ & 47 & $0.50(0.30)$ & $0.02(-0.12$ to 0.15$)$ \\
\hline
\end{tabular}

*Based on 107 patients in community hospital group and 58 in district general hospital group.

whether the service had been used ( $\kappa$ range $0.35-0.61$ ) but fair agreement over the frequency of use ( $\kappa$ range $0.14-0.41)$ with greater frequency of use according to the database for two resource categories, fewer for one, and concordance for the fourth.

The mean (standard deviation) costs per patient were similar for the community hospital and district general hospital groups (£7233 (£5031) v £7351 (£6229); mean difference - £118, 95\% confidence interval -1639 to 1403). The bootstrapped mean (standard deviation) cost was $£ 7243$ ( $£ 5026$ ) in the community hospital group and $£ 7375$ ( $£ 6225$ ) in the district general hospital group. On the basis of these simulations, the mean incremental cost effectiveness ratio suggests that community hospital care is slightly more effective and less costly than district general hospital care. The results of the bootstrap simulation show similar cost effectiveness between the two settings (fig 1). The cost effectiveness acceptability curve (fig 2) suggests that if decision makers' willingness to pay per QALY was $£ 10000$, then community hospital care would be cost effective in $51 \%$ of cases and that this would be increased to only $53 \%$ if the threshold for willingness to pay was raised to $£ 30000$.

Since the mean patient costs and QALYs for the community hospital group over the six months of follow-up were similar to those of the district general hospital group, providing post-acute care in a community hospital for this sample of older people is of similar cost effectiveness to post-acute care in a district general hospital.

\section{Sensitivity analyses}

Several sensitivity analyses were carried out to investigate the robustness of these results, concentrating on the main cost drivers of readmission to hospital and use of institutional care. The sensitivity analyses for institutional care involved varying the magnitude of patients' financial contributions for those who answered that they contributed to the cost of their care. When patients contributed the total cost of their care, the mean costs per patient for both arms were reduced and community hospital care remained a less expensive strategy, although the difference

Table 2 Use of health and social care resources during six months' follow-up of older patients admitted to a community hospital or district general hospital for rehabilitation

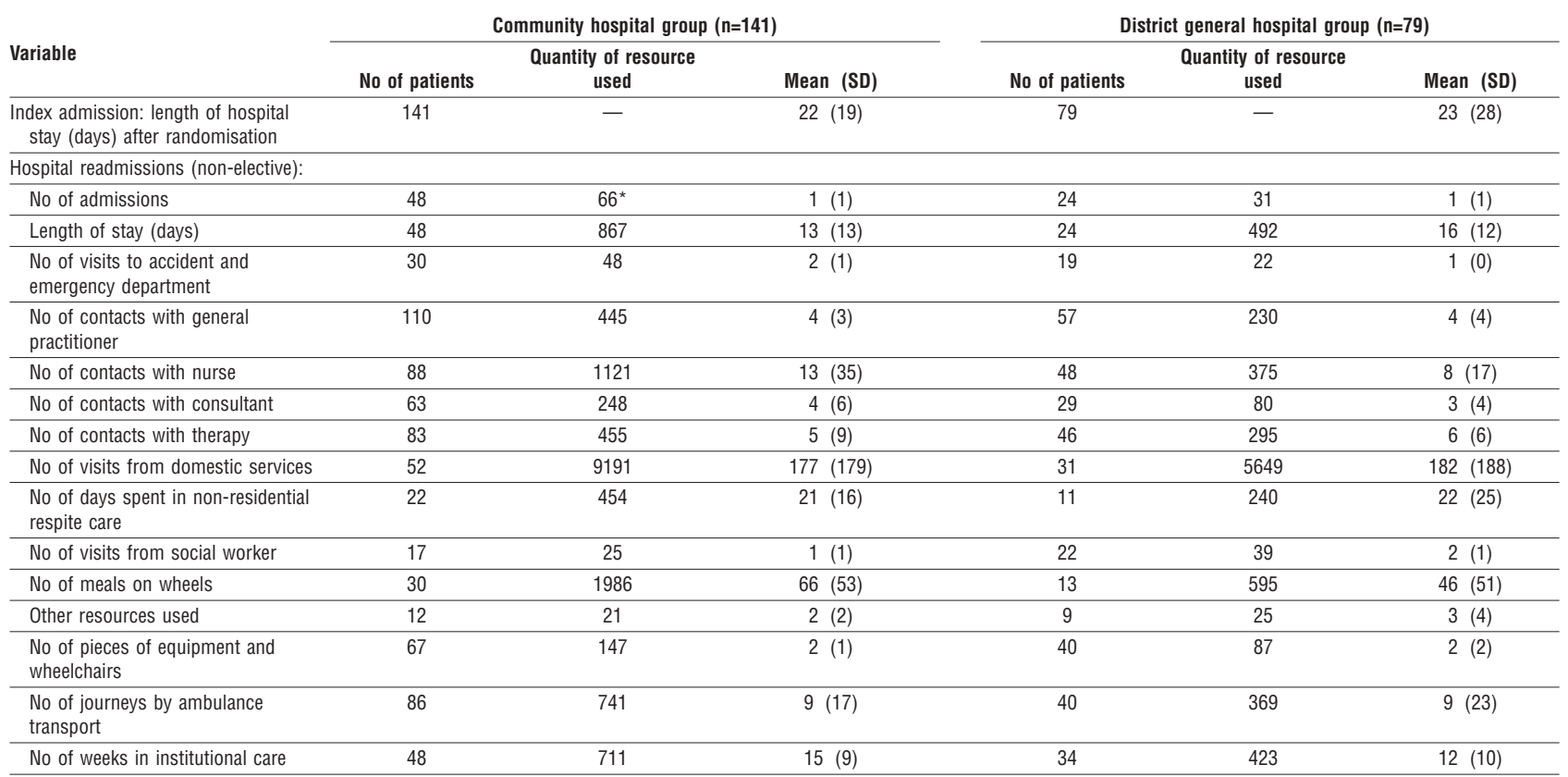

*Excludes three episodes that were deemed to be non-elective, but were recorded as a length of stay of 0 in patient administration system. These episodes were included in the cost analysis. Also excludes three patients who recorded five readmissions on the questionnaire that were at hospitals in areas outside those covered by the patient administration system. These readmissions were included in the cost analysis. 


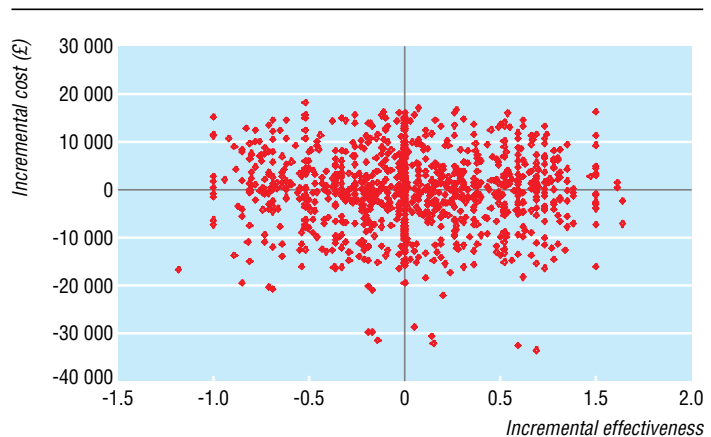

Fig 1 Representation of cost effectiveness plane on basis of bootstrap simulation (based on 1000 replications)

was non-significant (table 3). When institutional care was paid entirely by the public sector, the mean costs per patient increased relative to baseline but remained similar for the two groups.

In a further sensitivity analysis, the costs of episodes of hospital readmissions were calculated using health resource group costs only (that is, excluding the costs of any extended hospital stay). Although the mean costs per patient fell in both groups, community hospital care remained non-significantly less costly. The daily rate for the community hospital was varied using two scenarios. In the first scenario the rate was set at 10\% higher than that of the district general hospital. The difference in the mean costs per patient between the two groups narrowed relative to baseline, but the costs at the community hospital were marginally higher. When the daily rate for the community hospital was set at $20 \%$ above that of the district general hospital, the mean costs per patient in the community hospital arm was greater. In the final sensitivity analyses the daily rate for the district general hospital was reduced by $10 \%$ and $20 \%$. In both cases the mean costs incurred by patients in the community hospital arm were statistically non-significantly higher than those in the district general hospital group.

\section{Discussion}

The health outcomes and costs associated with community hospital care for older people in need of post-acute rehabilitation are similar to those of a department for the care of elderly people in a district general hospital. The improvement, albeit statistically non-significant, in quality adjusted life years for the community hospital group at six months was consistent with the greater independence experienced by this group, as measured

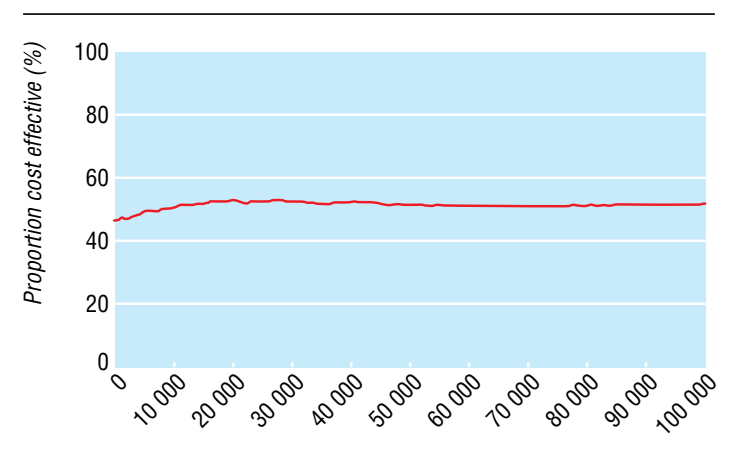

Willingness to pay (£) per quality adjusted life years

Fig 2 Cost effectiveness acceptability curve for older patients admitted to a community hospital compared with a district general hospital for rehabilitation (based on 1000 replications)
Table 3 Mean cost per older patient admitted to community hospital or district general hospital for rehabilitation and results of sensitivity analyses

\begin{tabular}{|c|c|c|c|}
\hline \multirow[b]{2}{*}{ Variable } & \multicolumn{3}{|c|}{ Mean cost per patient } \\
\hline & $\begin{array}{c}\text { Mean (SD) cost } \\
(£) \text { in } \\
\text { community } \\
\text { hospital group }\end{array}$ & $\begin{array}{l}\text { Mean (SD) cost } \\
(£) \text { in district } \\
\text { general hospital } \\
\text { group }\end{array}$ & $\begin{array}{c}\text { Mean }(95 \% \mathrm{Cl}) \\
\text { difference }(£)\end{array}$ \\
\hline Base case & 7233 (5031) & 7351 (6229) & $\begin{array}{c}-118 \\
(-1639 \text { to } 1403)\end{array}$ \\
\hline \multicolumn{4}{|l|}{ Sensitivity analyses: } \\
\hline $\begin{array}{l}100 \% \text { patient contributions } \\
\text { towards cost of institutional } \\
\text { care }\end{array}$ & 6294 (4369) & 6776 (5843) & $\begin{array}{c}-482 \\
(-1973 \text { to } 1009)\end{array}$ \\
\hline $\begin{array}{l}\text { Zero patient contributions } \\
\text { towards cost of institutional } \\
\text { care }\end{array}$ & 7636 (5516) & 7598 (6504) & $\begin{array}{c}38 \\
(-1593 \text { to } 1669)\end{array}$ \\
\hline $\begin{array}{l}\text { Using health resource } \\
\text { group costs only to } \\
\text { calculate cost of } \\
\text { readmissions }\end{array}$ & 6732 (4713) & 6738 (5873) & $\begin{array}{c}-6 \\
(-1435 \text { to } 1423)\end{array}$ \\
\hline $\begin{array}{l}10 \% \text { increase in community } \\
\text { hospital daily rate relative to } \\
\text { district general hospital } \\
\text { daily rate }\end{array}$ & 7472 (5182) & 7453 (6290) & $\begin{array}{c}19 \\
(-1625 \text { to } 1663)\end{array}$ \\
\hline $\begin{array}{l}20 \% \text { increase in community } \\
\text { hospital daily rate relative to } \\
\text { district general hospital } \\
\text { daily rate }\end{array}$ & 7750 (5367) & 7572 (6370) & $\begin{array}{c}178 \\
(-1413 \text { to } 1770)\end{array}$ \\
\hline $\begin{array}{l}10 \% \text { reduction in district } \\
\text { general hospital daily rate }\end{array}$ & 7128 (4983) & 7080 (6073) & $\begin{array}{c}48 \\
(-1447 \text { to } 1543)\end{array}$ \\
\hline $\begin{array}{l}20 \% \text { reduction in district } \\
\text { general hospital daily rate }\end{array}$ & 7022 (4939) & 6808 (5926) & $\begin{array}{c}214 \\
(-1258 \text { to } 1685)\end{array}$ \\
\hline
\end{tabular}

$£ 1.0(€ 1.46 ; \$ 1.8)$

by the Nottingham extended activities of daily living scale in the associated randomised controlled trial. ${ }^{10}$

A 2001 survey identified 471 community hospitals in the United Kingdom providing over 18000 beds and offering a range of inpatient and outpatient services. ${ }^{8}$ Community hospitals therefore comprise an important component of health care, particularly for older people who are the main users. ${ }^{20}$ Despite the long history of community hospital care, well carried out evaluation studies are scarce. Resource studies have investigated changes in district general hospital use associated with community hospital care by comparing patients in areas with and without access to community hospitals. ${ }^{21-23}$ This is not an ideal method, however, because essentially two different settings (rural and urban) are under comparison, and there are confounding factors with potential to influence bed use and length of stay. The renewed interest in community hospitals in the context of providing intermediate care ${ }^{7}$ has highlighted the weakness in the evidence base. The results of our cost effectiveness study of community hospital care are applicable to one function, post-acute rehabilitation for older people, and one type of community hospital, a locality or primary care trust based community hospital primarily providing an intermediate care service.

The strengths of our study are its randomised design with six months of follow-up. The economic perspective has been a whole systems one of health and social care costs combined over the six months of the study but excluded the issue of apportioning costs for informal carer burden. The carer burdens reported in the clinical outcomes study were, however, similar between the community hospital and control groups. ${ }^{10}$

Our study has some limitations. Firstly, it is a single site evaluation and represents only one of several possible models offered by community hospitals. ${ }^{10}$ Ideally our findings should be confirmed in other sites. Secondly, the mean age of the study population was 85 years, some of whom had cognitive impairment. The reliability of recall for events and services 
received over the six months is therefore a concern. This was mitigated by, whenever possible, interviewing patients in the presence of their carers. Furthermore, information on hospital stay and hospital readmissions (a major cost driver) was obtained from the hospital patient information system. We also checked for reliability of reported information on some community health and social services received against a community database and found moderate agreement. Finally, we extrapolated the health outcome and resource use data at six months to one year figures on the basis that these values were maintained during the remainder of the year. In reality these figures probably changed.

The results of our study suggest that using this locality based model of community hospital care for this group of patients is of similar cost effectiveness to district general hospital care provided in a department for the care of elderly people. A community hospital service similar to the one studied may be reasonably expected to reduce pressure on a district general hospital service by releasing beds. This conclusion, in conjunction with the favourable results from the associated clinical outcomes, ${ }^{10}$ and qualitative study, ${ }^{24}$ suggests that post-acute rehabilitation provided by a community hospital can be an effective model for intermediate care.

We thank our colleagues in the elderly care department, Bradford Teaching Hospitals NHS Foundation Trust; the managers and staff at Shipley community hospital; the case management team of north Bradford primary care trust; colleagues in the following organisations involved in the study: north Bradford primary care trust, Bradford Community NHS Trust, Bradford health authority, Bradford social services department, University of Bradford, York Health Economics Consortium at the University of York. The grant application signatories were JY, NS, KL, AF, and Angela Clegg. The trial steering group and project group members were JY, AF, NS, Susan Ince, Laura Hibbs, Angela Clegg, Joy Warburton, Jackie Hansford, Anne McAdam, Karen Mallinder, KL, JO'R, and JG. The study research team included Linda Dobrzanska, Helen Wright, Emma Tanner, Karen Mallinder, and JG.

Contributors: JO'R was responsible for the economic evaluation, drafting the paper, and intellectual content of the paper. KL was responsible for supervising the economic evaluation, drafting the paper, and the intellectual content of the paper, and was also a member of the steering group; she is guarantor. JY conceived the trial on which the economic evaluation is based; obtained funding; designed, initiated, and coordinated the study; and was responsible for analysis and interpretation of data, drafting the paper, and the intellectual content of the paper. AF obtained funding and designed, initiated, and coordinated the trial. JG managed the trial and was involved in collecting, collating, and analysing the data. NS was a

\section{What is already known on this topic}

Community hospitals are a long established component of healthcare provision in England

Previous health economic studies of community hospital care have been methodologically weak and difficult to interpret

\section{What this study adds}

A locality based community hospital is as cost effective as a district general hospital for post-acute care of older people member of the steering group, contributed to the study design, obtained funding, and was responsible for the qualitative component of the study.

Funding: Health Foundation.

Competing interests: JY and JG have worked in the community hospital involved in this study.

Ethical approval: This study was approved by the research ethics committee of Bradford Hospitals NHS Foundation Trust.

1 Department of Health. Creating a patient-led NHS: delivering the NHS improvement plan. Dondon:

Department of Health. The NHS plan. London: DoH, 2000.

3 Griffiths P, Wilson-Barnet J, Richardson G, Spilsbury K, Miller F, Harris R. The effectiveness of intermediate care in a nursing-led in-patient unit. Int J Nurs Stud 2000;37:153-61

4 Steiner A, Walsh B, Pickering RM, Wiles R, Ward J, Brooking J. Therapeutic nursing or unblocking beds? A randomised controlled trial of a post-acute intermediate care unit. BMJ 2001;322:453-60.

5 Griffiths P, Harris R, Richardson G, Hallett N, Heard S, Wilson-Barnett J. Substitution of a nurse led inpatient unit for acute services: a randomised controlled trial of outcomes and costs of nurse-led intermediate care. Age Ageing 2001:30:483-8.

6 Shepperd S, Iliffe S. Hospital at home versus in-patient hospital care. Cochrane Database Syst Rev 2005;(3):CD000356.

7 Meads G. Rediscovering community hospitals. Br J Gen Pract 2001;51:91-2

8 Seamark D, Moore B, Tucker H, Church J, Seamark C. Community hospitals for the new millennium. BrJ Gen Pract 2001;51:125-7.

9 Department of Health. Our health, our care, our say: a new direction for community services. Jan 2006: Cm 6737. Ch 6 para 6.43.

10 Green J, Young J, Forster A, Mallinder K, Bogle S, Lowson K, et al. Effects of locality based community hospital care on independence in older people needing rehabilitation: randomised controlled trial. BMJ 2005;331:317-22.

11 Brooks R. EuroQol: the current state of play. Health Policy 1996;37:53-72.

12 EuroQol EQ-5D user guide. Version A (6/96). www.euroqol.org (accessed 5 July 2006).

13 Chartered Institute of Public Finance and Accountancy. The health service financial data base and comparative tool 2002. London: CIPFA, 2002.

14 Department of Health Reference costs 2002 Leeds: $\mathrm{DoH}, 2002$

15 Netten A, Curtis L. Unit costs of health and social care 2002. Canterbury, Kent: Personal Social Services Research Unit, University of Kent at Canterbury, 2002

16 Hodkinson HM. Evaluation of a mental test score for assessment of mental impairment in the elderly. Age Ageing 1972;1:233-8.

17 Collin C, Wade DT, Davies S, Horne V. The Barthel ADL index: a reliability study. Int Disabil Stud 1988;10:61-3.

18 Briggs AH, Wonderling DE, Mooney CZ. Pulling cost-effectiveness analysis up by its bootstraps: a non-parametric approach to confidence interval estimation. Health Econ 1997;6:327-40.

19 Briggs AH, Mooney CZ, Wonderling DE. Constructing confidence intervals for cost-effectiveness ratios: an evaluation of parametric and non-parametric techniques cost-effectiveness ratios: an evaluation of parametric and

20 Young J, Donaldson K. Community hospitals and older people. Age Ageing 2001;30(suppl 3):7-10.

21 Baker JE, Goldacre M, Muir-Gray JA. Community hospitals in Oxfordshire. J Epidemiol Community Health 1986;40:117-20.

22 Hine C, Wood VA, Taylor S, Charny M. Do community hospitals reduce the use of district general hospital inpatient beds? J R Soc Med 1996;89:681-7.

23 Cook PJ, Porter L. Community hospitals and district general hospital medical bed use by elderly people: a study of 342 general practitioner beds in Oxfordshire. Age Ageing 1998;27:357-61.

24 Small N, Green J, Spink J, Forster A, Lowson K, Young J. The patient experience of community hospital-the process of care as a determinate of satisfaction. J Eval Clin Pract 2006:doi:10.1111/j.1365-2753. 2006.00653x.

(Accepted 22 May 2006)

doi $10.1136 /$ bmj.38887.558576.7C

York Health Economics Consortium, University of York, Heslington, York YO10 $5 \mathrm{NH}$

Jacqueline O'Reilly consultant

Karin Lowson deputy director

Academic Unit of Elderly Care and Rehabilitation, St Luke's Hospital, Bradford

John Young professor of elderly care medicine

Anne Forster reader

John Green research fellow

School of Health Studies, University of Bradford

Neil Small professor of health research

Correspondence to: K Lowson kvl2@york.ac.uk 